\title{
BREAKDOWN OF ROTATIONAL SYMMETRY AT SEMICONDUCTOR INTERFACES: A MICROSCOPIC DESCRIPTION OF VALENCE SUBBAND MIXING
}

\author{
S. Cortez, O. Krebs and P. Vorsin \\ Laboratoire de Physique de la Matière Condensée, Ecole Normale Supérieure \\ 24 rue Lhomond, Paris, France
}

\begin{abstract}
The recently discovered in-plane optical anisotropy of [001]-grown quantum wells offers a new theoretical and experimental insight into the electronic properties of semiconductor interfaces. We first discuss the coupling of $X$ and $Y$ valence bands due to the breakdown of rotation inversion symmetry at a semiconductor hetero-interface, with special attention to its dependence on effective parameters such as the valence band offset. The intracell localization of Bloch functions is explained from simple theoretical arguments and evaluated numerically from a pseudo-potential microscopic model. The role of envelope functions is then considered, and we discuss the specific case of non-common atom interfaces. Experimental results and applications to interface characterization are presented. These calculations give a microscopic justification, and establish the limits of the heuristic " $H_{\mathrm{BF}}$ " model.
\end{abstract}

PACS numbers: $78.20 . \mathrm{Fm}, 78.20 . \mathrm{Jq}$

\section{Introduction: breakdown of rotational symmetry at semiconductor interfaces}

It has been recently realized that the reduction of crystal symmetry at an abrupt hetero-interface may affect quite significantly the optical properties of semiconductor quantum wells, and in particular their isotropy with respect to the in-plane polarization of a light beam propagating parallel to the [001] growth axis. The cubic point group symmetry $T_{d}$ of the zinc-blende lattice is reduced to $C_{2 v}$ at an abrupt interface: it looses not only the translational invariance along the $z$-axis, but also an element of rotational symmetry, namely the invariance by the fourfold rotation inversion around the [001] direction. The arrangement of chemical bonds in the vicinity of an interface anion, shown in Fig. 1, illustrates this symmetry breakdown which has been until recently neglected in the classical envelope function theory (EFT). There are two bonds pointing forward along the [111] 


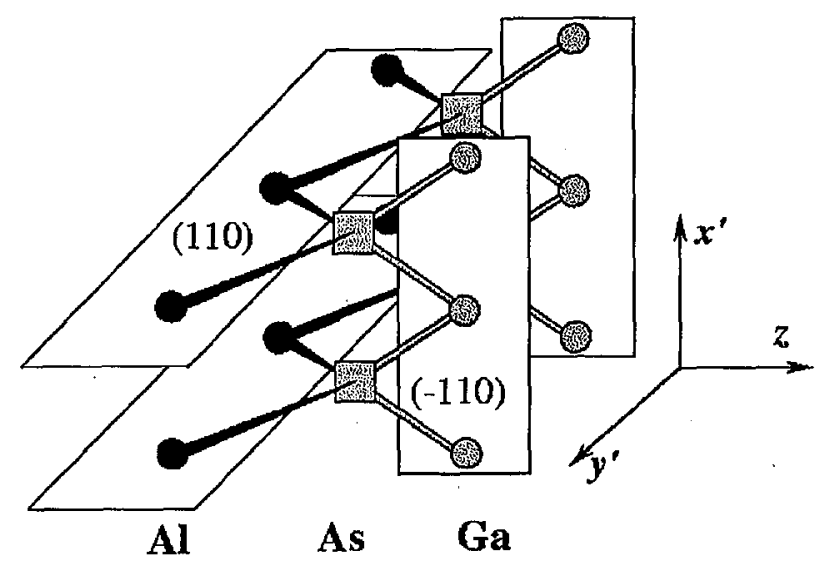

Fig. 1. Scheme of the geometrical arrangement of chemical bonds at a semiconductor interface.

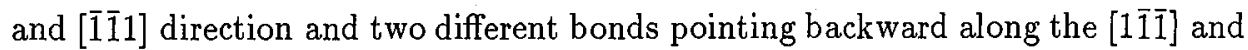
[i11] directions. The crucial point is that valence electrons lying on backward and forward bonds of an interface cell experience different potentials and interact with light polarized either along [110] or [i110]. In the absence of spin-orbit coupling, the threefold degeneracy of the bulk valence band with eigenfunctions $X, Y$, and $Z$ is lifted by the quantum confinement into three band with symmetries $X^{\prime}, Y^{\prime}$, and $Z$, where $X^{\prime}$ and $Y^{\prime}$ refer to $P$-type orbitals oriented along the [110] and [110] directions. These functions correspond to different representations of the group $C_{2 v}$, rotation anti-symmetric with respect to the planes $x^{\prime}=0$ or $y^{\prime}=0$. If the quantum well is symmetrical, its point group symmetry is $D_{2 d}$ and the $X^{\prime}$ and $Y^{\prime}$ representations are degenerate, but if it is asymmetrical, it retains the reduced symmetry $C_{2 v}$ of a single interface and the $X^{\prime}$ and $Y^{\prime}$ eigenstates are non-degenerate, which corresponds to in-plane anisotropy. As will be discussed further, reintroduction of the spin-orbit coupling at this point transforms the $X^{\prime}-Y^{\prime}$ splitting into a mixing of the heavy and light hole states in quantum well (QW) structures. Although this mixing had been discussed as early as 1985 in the tight-binding calculations of Schulmann and Chang [1], the topic has recently attracted much attention with the experimental discovery of the related giant optical anisotropy in quantum well structures [2] where the host materials do not share a common atom or non-common atom (NCA)-QWs, and the simultaneous development of envelope function theories [3-5] and atomistic (empirical $[2,6,7]$ or ab-initio [8]) calculations of this phenomenon. Optical anisotropy is sensitive to the details of interface structure, such as chemical nature of interface bonds, interface sharpness, etc... A detailed analysis of the dichroism and birefringence of quantum wells is therefore a new and major characterization tool to analyze the properties of semiconductor interfaces.

The purpose of this paper is to present recent theoretical developments of this topic, and experimental results illustrating the role of interfaces, and the effect of their detailed structure. The paper is organized as follow: in the second section, we discuss the main features of the zone-center Bloch functions, both from symmetry 
arguments, and numerically using the empirical pseudo-potential approach in the zero spin-orbit limit. Then we estimate the splitting of the eigenstates with $X^{\prime}$ and $Y^{\prime}$ symmetries in an interface unit cell, and introduce the envelope functions to obtain the splitting between corresponding quantum well states. The spin-orbit coupling is then introduced to recover the description of quantum well eigenstates in terms of mixed heavy- and light-hole states which allows the comparison with experiments. The last section is devoted to experimental analysis of quantum wells, with special emphasis on the properties of NCA systems, and on the effect of an external electric field, or "quantum confined Pockels effect" (QCPE).

\section{Microscopic wave functions in the zinc-blende semiconductors}

All of the following paragraph is known from experts of the different theoretical techniques, but apparently not from the semiconductor community at. large. Since the properties of microscopic wave functions is the key point of this paper, we first review a few general aspects. The $k \cdot p$ theory is based on the consideration of the symmetry of the zone-center eigenstates and a small number of interband matrix elements of the momentum: it generally ignores the problem of the intracell shape and localization of the Bloch functions (although, of course, the question would be implicitly solved if a complete basis were considered). However, when the crystal potential is modified on the scale of a unit cell, as for example in the case of an abrupt interface, it is intuitively evident that the matrix elements of the perturbation will be strongly dependent on the local properties of the Bloch functions. Therefore, it is crucial to use a realistic description of these functions and to examine their robustness against perturbations. Classical microscopic band structure calculations such as empirical tight-binding or pseudo-potentials may provide the necessary insight, but their predictions have to be examined critically. For instance, practical applications of the tight-binding models discussed in Ref. [9] or in classical textbooks are usually based on the assumption that the overlap between atomic functions centered on neighboring atoms is vanishingly small, in spite of significant intersite transfer matrix elements. This assumption implies obviously that the electronic density of any band has a minimum between two neighboring atoms, which, as discussed below, is incorrect. We of course do not intend here to criticize the tight-binding theory as a band structure parametrization method, but simply insist on the fact that the usual physical image of the microscopic wave functions is incorrect and may lead to qualitatively incorrect ideas. Conversely, the empirical pseudo-potential method is based on the Fourrier series expansion of the Bloch functions, and in principle its accuracy is limited only by the number of harmonics considered in the calculation, and the careful fitting of bulk band structure. In the following, we discuss microscopic wave functions obtained in the simplest pseudo-potential approach, ignoring spin-orbit interaction. The microscopic potential of a bulk semiconductor is expanded as

$$
V(\boldsymbol{r})=\sum_{\boldsymbol{G}} V_{\boldsymbol{G}} \exp (\mathrm{i} \boldsymbol{G} \boldsymbol{r}),
$$

where the coefficients $V_{\boldsymbol{G}}$ are fitted to reproduce the bulk band structure. The 


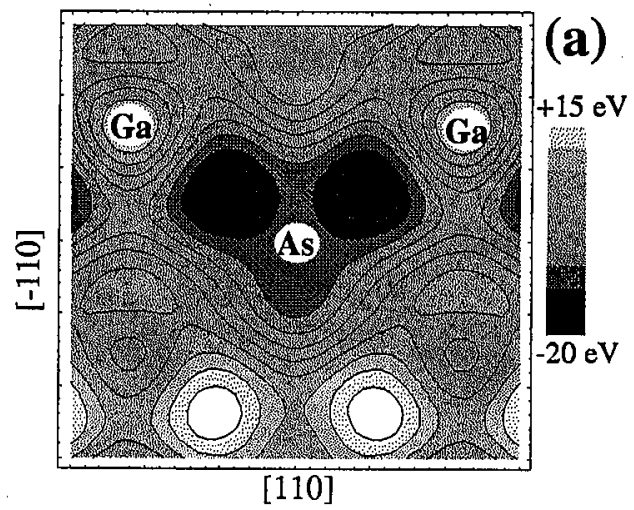

(b)

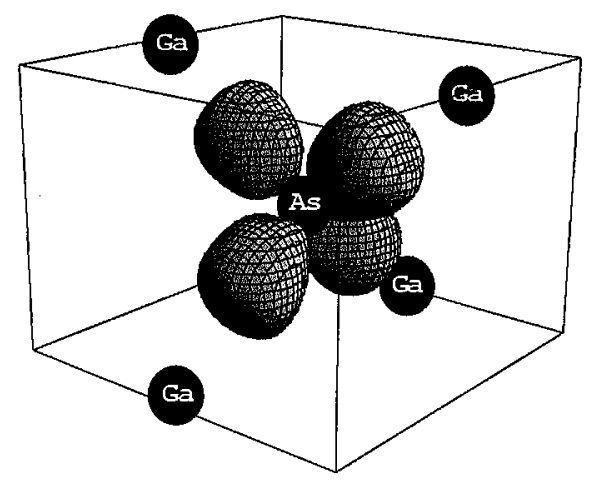

Fig. 2. (a) Microscopic empirical pseudo-potential of bulk GaAs in the plane (110). (b) Three-dimensional plot of the equipotential surface at $V(r)=-12 \mathrm{eV}$.

crystal Hamiltonian can easily be diagonalized in such a plane wave basis, yielding not only the correct energies, but also the microscopic shape of Bloch functions. These expansions, limited to three harmonics, correspond to a kinetic energy cut-off of $60 \mathrm{eV}$. While certainly insufficient to obtain details of the microscopic potential, this accuracy is good enough to describe the potential wells associated with the chemical bonds, and to provide a fair fit of the first valence and conduction bands throughout the Brillouin zone. Using pseudo-potential form factors given in Ref. [10], we show in Figs. 2-4 a few aspects of the microscopic potential and wave functions in GaAs. As can be seen in Fig. 2a, the potential in the plane $y^{\prime}=0$ which contains the forward bonds of Fig. 1 has two deep wells nearly at the center of the $\mathrm{Ga}$-As bonds. Typical figures are a well width of $L_{b}=2 \AA$ and a well depth of $20 \mathrm{eV}$. The equipotential surface $V(r)=-12 \mathrm{eV}$ shown in Fig. 2b illustrates this feature even more obviously. The isodensity surface of the $X^{\prime}$ and $Y^{\prime}$ functions displayed in Fig. 3 show that these wave functions are strongly localized, respectively in the backward and forward parts of the unit cell: $70 \%$ of the total $X^{\prime}$ (resp. $Y^{\prime}$ ) electronic density is contained in the displayed surfaces. Conversely, the $Z$ function (Fig. 4a) is equally distributed in the two parts of the cell. As for the 

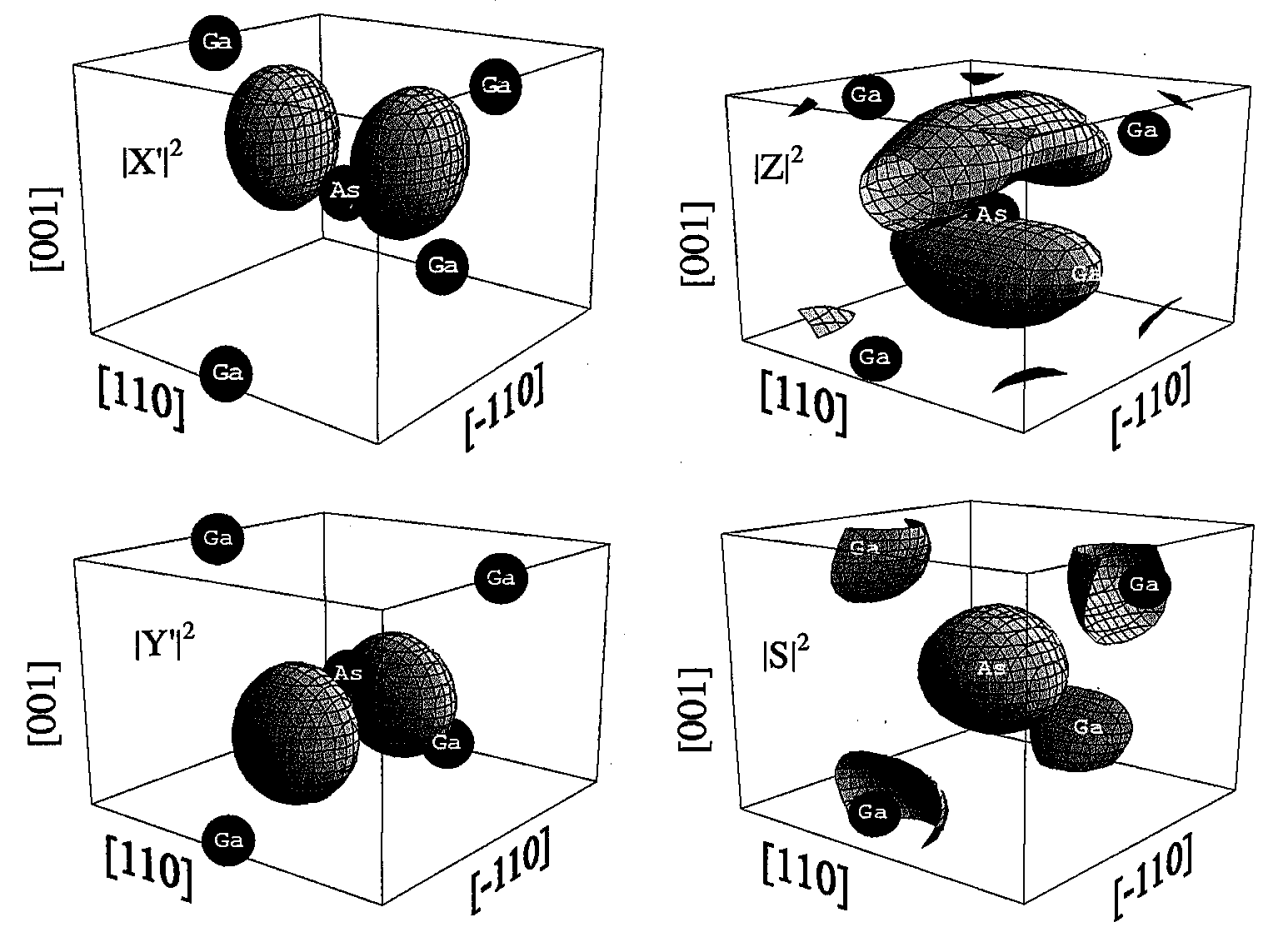

Fig. 3

Fig. 4

Fig. 3. Isodensity surfaces of the $X^{\prime}$ and $Y^{\prime}$ valence Bloch function at the zone center in bulk GaAs, showing their respective localization in the backward and forward half-cells. Fig. 4. Isodensity surfaces of the $Z$ valence Bloch function and $S$ conduction Bloch function at the zone center $\Gamma$ in bulk GaAs.

first conduction band shown in Fig. 4b, it does look like an antibonding combination of atomic $S$-like orbitals. These general features of the tetragonal bonds show little material-to-material variation: the main modification observed when using the pseudo-potential coefficients of CdTe is the increased ionicity, characterized by the fact that the electron density concentrates closer to the anion, both for the valence and conduction bands.

As the intracell localization of the valence states is of central interest, it is finally interesting to justify it on the sole basis of symmetry considerations. In the following, we shall decompose the kinetic energy contribution to the Hamiltonian as a sum of its contributions along the three orthogonal directions $x^{\prime}, y^{\prime}$, and $z$. We can formally express the kinetic energy of Bloch state along the direction $x^{\prime}$ as

$$
E_{c}^{x^{\prime}}=-\frac{\hbar^{2}}{2 m} \frac{\partial^{2}}{\partial_{x^{\prime}}^{2}} \approx-\frac{\hbar^{2}}{2 m} \frac{1}{d_{x^{\prime}}^{2}}
$$

where $d_{x^{\prime}}$ is a typical variation length of the Bloch function along the direction $x^{\prime}$. By definition, an eigenstate of symmetry $X^{\prime}$ is antisymmetrical with respect to the plane $x^{\prime}=0$ and symmetrical with respect to the plane $y^{\prime}=0$, while an eigenstate 
of symmetry $S$ or $Z$ is symmetrical with respect to both reflections. Hence $X^{\prime}(r)$ has a node in the plane $x^{\prime}=0$ which contains the two backward chemical bonds. If $X^{\prime}$ was confined equally in the four potential wells, it would experience a much larger (about four times larger) kinetic energy in the two backward wells where it has a node than in the two forward wells which are separated by the plane $x^{\prime}=0$, and where the $X^{\prime}$ symmetry can be accommodated with keeping a constant sign inside each well. The characteristic variation length of the $X^{\prime}$ function is of the order of only $d_{x^{\prime}}=L_{b} / 4=0.5 \AA$ in the backward region, while it would be of $d_{x^{\prime}}=L_{b} / 2=1 \AA$ in the forward wells. Corresponding kinetic energies are of the order of $20 \mathrm{eV}$ and $5 \mathrm{eV}$, respectively: kinetic energy acts as a strong potential repulsing the $X^{\prime}$ state out of the backward potential wells, and $Y^{\prime}$ states out of the forward wells. Another instructive way of understanding these localization properties was suggested by Khurgin [11] using a tight binding point of view: if one builds solutions of $X^{\prime}$ symmetry from atomic $X_{A}^{\prime}$ and $X_{C}^{\prime}$ functions, the intersite overlap (strong, albeit usually neglected in the band structure calculations) is binding in the forward region and anti-binding in the backward region.

To conclude this section, let us note that from the consideration of the orders of magnitude of the potential depth and length scale, the microscopic wave functions must be very robust against perturbations of the order of a fraction of $\mathrm{eV}$ such as a band offset, the spin-orbit interaction or a uniaxial stress: in general, first order perturbation theory should be enough to describe the effect of such perturbations. On a more intuitive ground, Figs. 2-4 suggest that the pseudo-potential coefficients are essentially related to the properties of the potential wells associated with individual bonds (including the admixture of all kinds of atomic states in the bond formation).

\section{Splitting of $X^{\prime}$ and $Y^{\prime}$ states at a GaAs-AlAs interface}

In order to study a GaAs-AlAs interface we shall first consider an interface unit cell centered on an As atom. The symmetry plane $x^{\prime}=0$ contains two backward GaAs bonds while the $y^{\prime}=0$ plane contains two forward AlAs bonds. In order to describe the microscopic potential $V(r)$ over the interface cell, we shall rely on the pseudo-potential approximation.

Let us call $V_{\mathrm{GaAs}}(r)$ and $V_{\mathrm{AlAs}}(r)$ the oscillatory parts of the bulk empirical pseudo-potentials of these two materials, which can be viewed as some "smoothed" approximation of the microscopic potential experienced by valence and conduction electrons. The potential near the interface depends on two different phenomena: (i) the physical nature of the bonds is different in the forward and backward regions, which can be characterized quantitatively by the difference of pseudo-potential coefficients $V_{3 a}, V_{3 c}, V_{4} \ldots$ of the semiconductors involved. This basically corresponds to a difference in the shape and depth of the potential wells corresponding to chemical bonds on both sides. (ii) There is a constant potential offset $\Delta V_{0}$ between the two materials, which includes the difference of average potential of the (supposedly spatially separated) materials and the charge transfer, essentially limited to the interface bonds, which produces an electrostatic potential step at the interface. When using bulk empirical pseudo-potentials, this constant offset needs to be adjusted by hand since it is not directly a bulk property of each 
material but rather a characteristic of the interface. We note that the interface charge transfer has the $C_{2 v}$ symmetry, hence it should also include a quadrupolar contribution. In the empirical pseudo-potential picture, the charge transfer ensures (to the first order of perturbation) the continuity of the 3-dimensional microscopic potential. In practice, we shall neglect the quadrupolar contribution. In this description, the microscopic potential $V(r)$ takes the value $V_{\mathrm{GaAs}}(\boldsymbol{r})$ when $r$ lies closer to a GaAs bond and $V_{\text {AlAs }}(r)+\Delta V_{0}$ when $r$ lies closer to an AlAs bond. One should note at this point that the microscopic potential proposed here is different from the microscopic potential used in most pseudo-potential calculations: the potential is associated with the closest chemical bond rather than to a combination of different atomic pseudo-potentials. We introduce the function

$$
\Delta V(r)=V_{\mathrm{AlAs}}(r)-V_{\mathrm{GaAs}}(r)+\Delta V_{0},
$$

which has the full $T_{d}$ symmetry of the bulk crystal (we neglect here the small difference in bulk lattice constants). $\Delta V(r)$ contains all the information on the change of microscopic potential between the two bulk materials. Therefore, the microscopic potential in the interface cell can be written as

$$
V(r)=V_{\mathrm{GaAs}}(r)+\alpha(r) \Delta V(r)
$$

where $\alpha(r)$ describes how the microscopic potential changes in the interface cell: in other words, it partitions the interface cell into GaAs-like and AlAs-like regions, with the correct $C_{2 v}$ symmetry. As long as the "bond potential well" picture discussed in the previous section prevails, what matters is that $\alpha(r) \approx 0$ close to the potential wells associated with the backward bonds and $\alpha(r) \approx 1$ close to the potential wells associated with the forward bonds. Clearly, the unidimensional step function $Y(z)$ is rather a poor approximation of $\alpha(r)$. The "waffle" step function $W_{1}(r)$ introduced by Foreman [5] and illustrated in Fig. $5 \mathrm{a}$ is obviously a much better approximation, and a smoother function $W_{2}(r)$ following the maxima of the pseudo-potential as illustrated in Fig. 5b, an even better approximation of the actual function $\alpha(r)$. We now have to estimate the splitting of the states of symmetry $X^{\prime}$ and $Y^{\prime}$ associated with the $C_{2 v}$ symmetry of the interface cell, namely:

$$
\left\langle X^{\prime}|\alpha(r) \Delta V(r)| X^{\prime}\right\rangle-\left\langle Y^{\prime}|\alpha(r) \Delta V(r)| Y^{\prime}\right\rangle,
$$

where the integration is performed over the interface unit cell. Note that this splitting corresponds to twice the $\mathrm{X}-\mathrm{Y}$ interface coupling $\langle X|\alpha(r) \Delta V(r)| Y\rangle$ that we previously evaluated in the $H_{\mathrm{BF}}$ model [3], since $X Y=(1 / 2)\left(X^{\prime 2}-Y^{\prime 2}\right)$. A fully numerical evaluation of this matrix element has already been attempted, but it essentially relies on our capacity to describe correctly the difference of pseudo-potential coefficients $\Delta V_{0}, \Delta V_{3 a}, \Delta V_{3 c} \ldots$ between AlAs and GaAs with a precision of the order of $10 \mathrm{meV}$, which seems extremely optimistic if one compares the pseudo-potential coefficients obtained in the literature for these materials. In the following, we propose a way to link the spatially dependent function $\Delta V(r)$ with effective parameters such as the band offsets $\Delta E_{v}$ used in the classical envelope function theory, and therefore simplify these matrix elements.

It follows from the "bond potential well" picture that the $X^{\prime}$ function entering the expression (5) is similar to the $X^{\prime}$ function of bulk GaAs and the $Y^{\prime}$ 

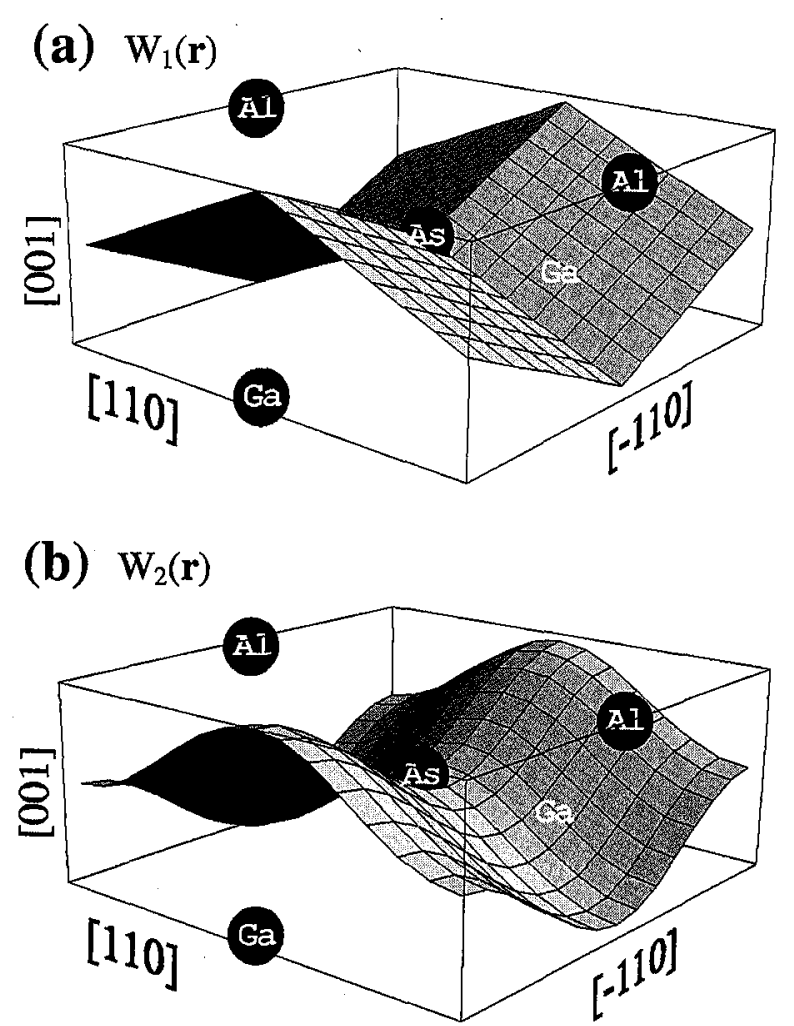

Fig. 5. Two kinds of "waffle" surfaces used in the calculation of the matrix elements to separate properly the backward and forward half-cells.

function close to that of bulk AlAs. We remind that the general features of these functions depend very little on the material. Also, if the localization of the microscopic function in the corresponding potential wells is strong enough, $\alpha(r)$ is close to zero in the region where $Y^{\prime}$ is non-vanishing, and close to 1 in the region where $X^{\prime}$ is non-vanishing. Within these approximations, which are discussed numerically in the following, the splitting approximates to $\left\langle X^{\prime}|\Delta V(r)| X^{\prime}\right\rangle$, which will be shown to be the valence band offset between the two materials. The correctness of this result is linked to the degree of localization of the $X^{\prime}$ and $Y^{\prime}$ functions with respect to the step change of the microscopic potential, and to the validity of this potential. In order to check this, we have calculated the degree of localization of the pseudo-potential functions in a bulk GaAs unit cell. In the following, all numerical values are based on the pseudo-potential form factors from Ref. [10]. We obtain a strong localization of the Bloch functions: $65 \%$ of the charge of the $X^{\prime}$ state is in the upper half-cell when partitioning the cell with a step function $Y(z)$, but the figure becomes $88 \%$ when using the "waffle" step function $W(r)$ between each half-monolayer. Therefore,

$$
\begin{gathered}
\left\langle X^{\prime}|\alpha(r)| X^{\prime}\right\rangle \approx\left\langle X^{\prime} \mid X^{\prime}\right\rangle=1 \text { hence }\left\langle X^{\prime}|\alpha(r) \Delta V(r)| X^{\prime}\right\rangle \approx\left\langle X^{\prime}|\Delta V(r)| X^{\prime}\right\rangle \\
\left\langle Y^{\prime}|\alpha(r)| Y^{\prime}\right\rangle \approx 0 \text { hence }\left\langle Y^{\prime}|\alpha(r) \Delta V(r)| Y^{\prime}\right\rangle \approx 0
\end{gathered}
$$


We now need to define properly the valence band offset between the two materials from a microscopic point of view, and to link it to the matrix element $\left\langle X^{\prime}|\Delta V(r)| X^{\prime}\right\rangle$. Let us consider two bulk unit cells of GaAs and AlAs far from the interface. They are described by two Hamiltonians which differ only by the potential $\Delta V(r)$ introduced previously

$$
H^{\mathrm{AlAs}}=H^{\mathrm{GaAs}}+\Delta V(r) .
$$

The band offset is the difference of energy of the zone center $(k=0)$ Bloch states of these two Hamiltonians. If the Bloch functions change very little from one material to the other, a first order perturbation will directly give the difference of energy between the Bloch states in Al̄̈s and GaAs

$$
\begin{aligned}
& E_{v}^{\mathrm{AlAs}}=E_{v}^{\mathrm{GaAs}}+\Delta E_{v} \approx E_{v}^{\mathrm{GaAs}}+\langle X|\Delta V(r)| X\rangle, \\
& E_{c}^{\mathrm{AlAs}}=E_{c}^{\mathrm{GaAs}}+\Delta E_{c} \approx E_{c}^{\mathrm{GaAs}}+\langle S|\Delta V(r)| S\rangle .
\end{aligned}
$$

In order to check numerically this general result, we first need to adjust the constant coefficient $\Delta V_{0}$ in (3) to reproduce correctly the valence band offset. By setting $\Delta V_{0}=-0.12 \mathrm{eV}$ and diagonalizing separately the two Hamiltonians, we can reproduce the physical offset $\Delta E_{v}=-0.562 \mathrm{eV}$, which lies within $10 \%$ of the first order approximation $\langle X|\Delta V(r)| X\rangle=-0.525 \mathrm{eV}$, where the function $X$ used is the Bloch state of bulk GaAs.Intuitively, we can say that the band offset viewed by a given Bloch state $|X\rangle$ is the average value of the microscopic function $\Delta V(r)$ in the region where $|X\rangle$ has its maximum of density. Combining the previous results, we can conclude that the energy splitting between $X^{\prime}$ and $Y^{\prime}$ states over an interface cell is the valence band offset, which is exactly the result first predicted in the $H_{\mathrm{BF}}$ formalism using projection operators [3]

$$
\left\langle X^{\prime}|\alpha(r) \Delta V(r)| X^{\prime}\right\rangle-\left\langle Y^{\prime}|\alpha(r) \Delta V(r)| Y^{\prime}\right\rangle=\Delta E_{v} .
$$

One can easily compare the above result with a fully numerical evaluation of the above matrix elements, using bulk GaAs Bloch states and expanding the function $\alpha(\boldsymbol{r}) \Delta V(r)$ in the special case of a waffle interface. It yields a splitting of $0.511 \mathrm{eV}$, in close agreement with the valence band offset. The crucial point is that even this fully numerical computation requires first to adjust properly the constant coefficient $\Delta V_{0}$. We can conclude that in a non self-consistent approach, the $X^{\prime}-Y^{\prime}$ splitting is essentially governed by the valence band offset at the interface, and not by the microscopic details of the change of potential between the two materials. Of course, the splitting might be reduced in the case of a smoothed interface potential, but this main feature will remain.

\section{Interface induced $X-Y$ mixing: the role of the envelope functions}

In order to fully understand the role of the interfaces on the $X-Y$ band mixing in more realistic situations, we shall consider now whole AlAs-GaAs based heterostructures, where bound states will be described by usual envelope functions. Similarly to the previous section, we now define a generalized step-function $\alpha(r)$ over the whole structure in order to write correctly the microscopic potential. More precisely, $\alpha$ is equal to 1 (resp. 0 ) inside each half-monolayer representing a plane of AlAs (resp. GaAs) chemical bonds, with discontinuities along waffle-like 
interfaces. Moreover, it is particularly interesting to consider an electric field $F$. applied along $z$ (specifically in the case of common atom systems) since it modifies the shape of the envelope function and therefore may act onto the interface induced mixing [12]. For this reason, we shall consider the general microscopic potential

$$
V(r)=V_{\mathrm{GaAs}}(r)+e F z+\alpha(r) \Delta V(r)
$$

and focus our attention on the states corresponding to $k_{\perp}=\left(k_{x}, k_{y}\right)=0$. As already mentioned, the Bloch functions change very little between two III-V materials, although the energy gaps and therefore the effective masses can be very different. The method used is to start from an envelope function theory in which we neglect the coupling introduced by $\alpha(r) \Delta V(r)$ between states of opposite symmetries such as $X$ and $Y$, treating it later as a perturbation. This Hamiltonian can be diagonalized by block in each subspace of symmetry $X, Y$ or combinations of $Z$ and $S$ (because of the $k \cdot \boldsymbol{p}$ coupling between these states). This problem was solved long ago in the framework of the classical envelope function theory. The upper valence states, we are interested in, are essentially of the form $f_{p} X_{v}, f_{q} Y_{v}$ where the $\left\{f_{p}\right\}$ are different envelope functions, plus a small contribution from higher $X_{c}$ and $Y_{c}$ bands of no interest here. The envelopes above already include the effect of the electric field. At this point, one should notice that a careful treatment yields exactly the same envelope functions $\left\{f_{p}\right\}$ for both $X$ and $Y$ symmetries. We shall now introduce the $X-Y$ coupling as a perturbation on the previous basis, that is evaluate the matrix elements

$$
\Delta_{X Y}^{p q}=\left\langle f_{p} X|\alpha(r) \Delta V(r)| f_{q} Y\right\rangle
$$

where the integration is performed over the whole crystal. In order to get some physical insight on this rather complicated function, we make use, as in the previous section, of the fact that $X Y=(1 / 2)\left(X^{\prime 2}-Y^{\prime 2}\right)$. Then, using appropriate (waffle-like) interfaces we partition the crystal in a stack of monolayers where the regions of space closer to a forward bond are well separated from those closer to a backward bond. It allows us to decompose (11) as

$$
\Delta_{X Y}^{p q}=\sum_{z_{i}} \frac{1}{2}\left(\left\langle f_{p} X^{\prime}|\alpha \Delta V| f_{q} X^{\prime}\right\rangle_{W_{i}}-\left\langle f_{p} Y^{\prime}|\alpha \Delta V| f_{q} Y^{\prime}\right\rangle_{W_{i}}\right)
$$

where the summation runs over the successive monolayers indexed by $z_{i}$, which can be chosen as the coordinates either of the anionic or of the cationic planes. The matrix elements in (12) with subscript " $W_{i}$ " are calculated over these monolayers centered on $z_{i}$. Due to the localization properties of the $X^{\prime}$ and $Y^{\prime}$ states discussed in Sec. 2, each term of (12) can be seen as the valence band potential averaged respectively over the forward ( $X^{\prime}$-terms) and backward ( $Y^{\prime}$-terms) bonds and weighted by the product of the envelope functions. This is precisely the intuitive idea which had been developed through the original $H_{\mathrm{BF}}$ model [3]. Now we will use the fact that the envelope functions vary slowly at the scale of the monolayers, so they can be factorized from each matrix element. However, we also have to keep in mind that they must be evaluated at different positions inside the same monolayer, depending on the average position of $X^{\prime 2}$ or $Y^{\prime 2}$. Without this precaution, it would appear some theoretical inconsistency related to the choice of anion-centered or cation-centered monolayers [13, 14]. For simplicity, we can 
assume quasi-germanium Bloch functions, and take the value of the envelopes at the center of each chemical bond, i.e. at $z_{i} \pm a / 8$. This approximation mainly neglects the bulk inversion asymmetry (BIA) which actually does not affect much the shape of $X^{\prime}$ or $Y^{\prime}$ as previously discussed. We then obtain the following:

$\Delta_{X Y}^{p q}=\sum_{z_{i}} \frac{a}{4}\left[\left(f_{p} f_{q}\right)_{z_{i}+a / 8}\left\langle X^{\prime}|\alpha \Delta V| X^{\prime}\right\rangle_{W_{i}}-\left(f_{p} f_{q}\right)_{z_{i}-a / 8}\left\langle Y^{\prime}|\alpha \Delta V| Y^{\prime}\right\rangle_{W_{i}}\right]$,

where the factor $a / 2$ has been introduced, because of the normalization of $X^{\prime}$ and $Y^{\prime}$ states over $a / 2$-thick monolayers, and of the envelope functions*. Taking into account the results of Sec. 3 and the properties of the function $\alpha$, Eq. (13) can be simplified to

$$
\Delta_{X Y}^{p q}=\frac{a}{4} \Delta E_{v} \sum_{z_{i}}\left[\left(\alpha f_{p} f_{q}\right)_{z_{i}+a / 8}-\left(\alpha f_{p} f_{q}\right)_{z_{i}-a / 8}\right] .
$$

Because of the sign reversal between forward $\left(z_{i}+a / 8\right)$ and backward $\left(z_{i}-a / 8\right)$ bonds, each term of the sum essentially cancels its appropriate neighbor, except for the small changes of the envelope function weights or the potential discontinuities. By developing the envelope functions at the first order in $(a / 4) \partial\left(f_{p} f_{q}\right) / \partial z$ and assuming that they vanish for large $|z|$ in AlAs barriers, one finally gets

$$
\Delta_{X Y}^{p q}=\frac{a}{8} \Delta E_{v} \sum_{z_{i}^{\prime}}(-1)^{i}\left(\alpha_{z_{i}^{\prime}+a / 8}-\alpha_{z_{i}^{\prime}-a / 8}\right) f_{p}\left(z_{i}^{\prime}\right) f_{q}\left(z_{i}^{\prime}\right) .
$$

Now the summation runs over all the successive atomic planes which are indexed by $z_{2 i-1}^{\prime}=z_{i}$ and $z_{2 i}^{\prime}=z_{i}+a / 4$. This expression shows that the $X-Y$ mixing term is the alternated sum of all the discontinuities between half-monolayers weighted by the values of the envelope functions. The sign dependence $(-1)^{i}$ which appears in (15) can be seen as the effect of opposite symmetry breakdowns localized either on anionic or cationic planes. It can be noticed that taking into account the first-order envelope function variations has resulted in a factor $1 / 2$, which represents the main difference with the original $H_{\mathrm{BF}}$ model [3]. Thanks to this improvement, the model is no longer dependent on the choice of the cells used to partition the crystal ${ }^{\dagger}$. Moreover, in the case we are considering here, it is clear that all the discontinuities occur on As planes, since the bonds on both sides of an $\mathrm{Al}$ or $\mathrm{Ga}$ plane are necessarily equivalent (i.e. $\mathrm{As}-\mathrm{Al}-\mathrm{As}$ or $\mathrm{As}-\mathrm{Ga}-\mathrm{As}$ ). It follows that the general expression (15) reduces to the sum over theses anionic planes, for which the factor $(-1)^{i}$ is constant and can then be omitted.

In order to get a first application of this result, let us consider a single AlAs-GaAs QW. It presents only two discontinuities $\left(\alpha_{z_{\mathrm{L}}+a / 8}-\alpha_{z_{\mathrm{L}}-a / 8}=\right.$ $\left.-\left(\alpha_{z_{\mathrm{R}}+a / 8}-\alpha_{z_{\mathrm{R}}-a / 8}\right)=1\right)$, respectively situated on the As plane of the left $\left(z_{\mathrm{L}}\right)$ and right $\left(z_{\mathrm{R}}\right)$ interface. The $X-Y$ mixing becomes

$$
\Delta_{X Y}^{p q}=\frac{a}{8} \Delta E_{v}\left[f_{p}\left(z_{\mathrm{L}}\right) f_{q}\left(z_{\mathrm{L}}\right)-f_{p}\left(z_{\mathrm{R}}\right) f_{q}\left(z_{\mathrm{R}}\right)\right] .
$$

*We suppose that the envelope functions are normalized according to $\int f_{p}^{2}(z) \mathrm{d} z=1$.

${ }^{t}$ There is still arbitrariness in associating the $X^{\prime}$ state to the forward bonds (i.e. bonds from anion to cation in the $z$ direction) since in $D_{2 d}$ symmetry the directions [110] and [110] cannot be distinguished. It means that the matrix elements $\Delta_{X Y}^{p q}$ have not an absolute sign, but making the opposite choice would suppose to have rotated the $x, y$ axes by $\pi / 2$. 
It shows that in the case of a perfect common atom quantum well under zero electric field, for which the envelope functions are either even or odd, there is an exact compensation of the two interfaces for the states of the same symmetry. The interface coupling is non-zero only for states of opposite parity, which is consistent with the $D_{2 d}$ symmetry of such a system. In theory, an AlAs-GaAs quantum well cannot present optical anisotropy unless the roto-inversion symmetry of the structure is broken by an external perturbation like an electric field $F$ applied along the growth direction $z$. However, there is no direct coupling between $\left|f_{p} X\right\rangle$ and $\left|f_{q} Y\right\rangle$ induced by such a field. This result is obviously expected in the quasi-Ge approximation and remains actually valid when we include some ionicity in the localization properties of the Bloch functions. In order to check that point, we first use the expression (14) with $e F z$ instead of $\alpha \Delta V$, where the values of the function $z f_{p} f_{q}$ are now taken at the positions $z_{i} \pm a / 8$ shifted by $\pm \delta$ in order to include the slight deformation of the Bloch functions $X^{\prime}$ and $Y^{\prime}$ toward the anions As. Since the function $z f_{p} f_{q}$ vanishes rapidly inside the barriers, we obtain

$$
\left\langle f_{p} X|e F z| f_{q} Y\right\rangle=\frac{e F a}{8}\left[\frac{a}{4} \sum_{z_{i}^{\prime}}(-1)^{i} \frac{\partial\left(z f_{p} f_{q}\right)}{\partial z}\left(z_{i}^{\prime}\right)+2 \delta \sum_{z_{i}^{\prime}} \frac{\partial\left(z f_{p} f_{q}\right)}{\partial z}\left(z_{i}^{\prime}\right)\right] \approx 0 .
$$

The first term in (17) corresponds to the quasi-Ge treatment of $e F z$. It consists like (15) in an alternated sum over the cationic and anionic planes which experience the difference of a verage potential $e F a / 4$ between successive bonds, and approximates to zero at the first order in $a / L$ (where $L$ is the typical extension of the envelopes). The second term which is linear in $\delta$ represents the contribution of the ionicity of the Bloch functions (due to the BIA) and vanishes to the first order in $\delta / a$. As a result, the valence band mixing which can be generated in that case comes only from the deformation of the envelopes by the electric field which changes the respective weights of the interface contributions in (16). It gives rise to a completely original electro-optic effect known as the "quantum confined Pockels effect" (QCPE) [12], first observed by Kwok et al. [15] in electro-luminescence experiments. Although there is also a BIA contribution to the Pockels effect through the $k_{z}$ dependence of the optical matrix element [16], a remarkable feature of the QCPE is that it does not rely on the inversion asymmetry of the bulk crystal, and would be observable in QWs made out of centrosymmetric hosts like Ge and $\mathrm{Si}$. The concepts introduced in this section to calculate the matrix elements $\Delta_{X Y}^{p q}$ and specifically the expression (15) are very general and will be extended to the non-common atom interfaces and to the coupling between heavy and light holes.

\section{The case of interfaces between hosts having non-common atom}

As first pointed out in Ref. [3], the physics involved in the description of NCA interfaces (a situation encountered in the technologically important InAs-GaSb and (InGa)As-InP systems) shows striking differences with the case of common anion interfaces: in NCA systems, the interfaces involve specific chemical bonds which do not exist in either of the hosts, and in general, the specific bonds formed at the first and second interfaces of a quantum well differ, so that, even in absence of external electric field, the QW retains the low symmetry of a single interface. 
In addition, these interface bonds usually undergo considerable strain. We shall consider as an example in this section an interface between InAs and GaSb having the following sequence: ...-In-As-Ga-Sb. . Although InAs and $\mathrm{GaSb}$ are almost lattice-matched, the structure contains a highly strained half-monolayer composed of As-Ga chemical bonds, all lying in the same plane, say $y^{\prime}=0$. Obviously, the potential wells associated with these bonds differ from the neighboring "bulk-like" potential wells by their native pseudo-potential coefficients, and by the effect of strain. As found numerically in $a b$-initio calculations [8], the latter effect is probably the dominating one. It can be understood in terms of the kinetic energies of the different valence states. In the present example, the As-Ga half-monolayer experiences a $15 \%$ tensile strain along the $x^{\prime}$ axis. Let us formally consider the effect of such a deformation $\varepsilon$ on the Bloch states of a unit cell of bulk GaAs experiencing the same strain. The characteristic variation length $d_{X^{\prime}}$ of Bloch functions along $x^{\prime}$ depends linearly on the strain, therefore the kinetic energy contribution along this direction shall be reduced by $30 \%$. Since the valence state of symmetry $X^{\prime}$ has a node in the plane $x^{\prime}=0$, most of its kinetic energy is due to the $x^{\prime}$ contribution, unlike the states $Y^{\prime}$ or $Z$ (the latter being mixed with $S$ states by this uniaxial strain). The total kinetic energy of unstrained $\mathrm{GaAs}$ valence states calculated by pseudo-potentials being of $16 \mathrm{eV}$, we can expect the $X^{\prime}$ state kinetic energy to be shifted typically by an amount of $-3 \mathrm{eV}$ with respect to unstrained bulk GaAs. Of course, this huge effect is compensated in part by charge transfers and by the associated compressive strain along the $z$ direction, but it is clear that it contributes by an enormous amount to the $X^{\prime}-Y^{\prime}$ energy splitting.

We shall not attempt here to guess an approximate geometry and microscopic potential $V(r)$ at the interface to compute the splitting between $X^{\prime}$ and $Y^{\prime}$ but rather show how effective parameters can be introduced to characterize an interface. Following the ideas of Sec. 3, we can define an average valence potential for the states of symmetry $X^{\prime}$ in this half-monolayer by taking the matrix element $V_{\mathrm{GaAs}}=\left\langle X^{\prime}|V(\boldsymbol{r})| X^{\prime}\right\rangle_{1 / 2 \text { cell }}$ over the region of space closer to the GaAs bonds, that is in practice between two waffle interfaces. The Bloch state $X^{\prime}$ formally used in this matrix element is similar to the Bloch states of InAs and GaSb since their geometrical features are essentially governed by the length of the chemical bond along $x^{\prime}$ which is imposed by the substrate, the direction $z$ having only a very secondary effect here. One should note at this point that although we may get a physical idea of the shape of the bond quantum wells associated to the GaAs half-monolayer, that is the pseudo-potential coefficients of the strained bond, we have no mean to fit properly the constant $\Delta V_{0}$ in this part of the crystal since it cannot be adjusted with an experimental band offset. Hence, evaluating this potential is absolutely impossible without performing detailed self-consistent calculation because of the large charge transfer effects and the modifications of energy levels due to the strain. Therefore, we shall consider it as a characteristic of the interface, to be matched with experiments.

A single NCA interface can be viewed as two consecutive common anion and common cation interfaces with opposite orientations. There is therefore two potential discontinuities at the positions $z_{\text {int }} \pm a / 8$, which contribute to $\Delta_{X Y}^{p q}$ with an opposite sign (see Eq. (15)) yielding a $X-Y$ coupling of the following form: 


$$
\begin{aligned}
& \Delta_{X Y}^{p q}=\left\langle f_{p} X|V(r)| f_{q} Y\right\rangle= \\
& \quad \frac{a}{8} f_{p}\left(z_{\mathrm{int}}\right) f_{q}\left(z_{\mathrm{int}}\right)\left[\left(V_{\mathrm{InAs}}-V_{\mathrm{GaAs}}\right)-\left(V_{\mathrm{GaAs}}-V_{\mathrm{GaSb}}\right)\right] .
\end{aligned}
$$

The interface mixing is proportional to the difference of valence band offset of the two consecutive virtual common atom interfaces, $V_{\mathrm{InAs}}$ and $V_{\mathrm{GaSb}}$ being the valence band potential in the two bulk materials. Since the high interface strain can move the $X^{\prime}$ valence energy level $V_{\mathrm{GaAs}}$ far from the valence energies of both InAs and $\mathrm{GaSb}$, we can expect this interface coupling to be much larger than in the case of a simple common atom interface, and, of course, to depend drastically on the growth sequence. Instead of using the average values of the valence energy of each half-monolayer, it makes sense to write the $X-Y$ mixing in terms of the two parameters $d V_{\text {int }}=V_{\mathrm{InAs}}-V_{\mathrm{GaAs}}$ and $\Delta E_{v}=V_{\mathrm{GaSb}}-V_{\mathrm{InAs}}$, where $d V_{\text {int }}$ has to be fitted to experimental data and $\Delta E_{v}$ is the valence band offset. Hence

$$
\Delta_{X Y}^{p q}=\frac{a}{8} f_{p}\left(z_{\text {int }}\right) f_{q}\left(z_{\text {int }}\right)\left(2 d V_{\text {int }}+\Delta E_{v}\right) .
$$

Experimentally, in the case of (InGa)As-InP quantum wells [17], the typical order of magnitude for these interface potentials is $d V_{\text {int }} \approx 1 \mathrm{eV}$, which leads to matrix elements of a few meV. This means that in the zero spin-orbit limit, the optical absorption spectra of such heterostructures should show a polarization dependent optical gap. As we shall now point out, this effect is strongly modified by the large mixing of valence states due to the spin-orbit coupling.

\section{Introduction of the spin-orbit coupling and calculation of optical spectra}

Although the analysis of symmetry breakdown that we have discussed above describes correctly the microscopic effect of interfaces, it misses the introduction of spin-orbit coupling effects. The matrix elements due to the interface symmetry breakdown (a few meVs) are about two orders of magnitude smaller than the spin-orbit coupling constant (a few hundred meVs). Therefore, spin-orbit interaction cannot be treated as a perturbation over the states of symmetry $X^{\prime}, Y^{\prime}$, and $Z$. Instead, the interface effect can be conveniently treated as a perturbation in the traditional heavy and light hole basis that diagonalizes the spin-orbit interaction. The top valence band zone center states of a bulk material: $\mathrm{H}^{+}, \mathrm{L}^{+}, \mathrm{H}^{-}, \mathrm{L}^{-}$ are expressed as combinations of $X, Y$, and $Z$ Bloch states with appropriate spins, for example

$$
H^{+}=\frac{1}{\sqrt{2}}(X+\mathrm{i} Y) \uparrow, \quad L^{-}=\frac{1}{\sqrt{6}}(X-\mathrm{i} Y) \uparrow-\sqrt{\frac{2}{3}} Z \downarrow .
$$

Following the procedure of Sec. 4, we can diagonalize the Hamiltonian $H=E_{c}+$ $V(r)+H_{\text {s.o. }}$ in each symmetry block (with the appropriate conduction bands to take into account the $\boldsymbol{k} \cdot \boldsymbol{p}$ coupling) and then treat the inter-block matrix elements as first order perturbations. This means that we need to evaluate matrix elements between states which are essentially of the form $\left|f_{H_{p}} H^{+}\right\rangle$and $\left|f_{L_{q}} L^{-}\right\rangle$, plus small contributions from higher zone center states essentially negligible in the context of interface couplings. If we develop the Bloch function part of the matrix element $M_{H_{p}^{+} L_{q}^{-}}=\left\langle f_{H_{p}} H^{+}|V(\boldsymbol{r})| f_{L_{q}} L^{-}\right\rangle$, the only non-vanishing contribution will be of 
the form $\mathrm{i} / \sqrt{3}\langle X|V(r)| Y\rangle$, which means that the heavy-light hole coupling is directly proportional to the $X-Y$ coupling calculated with appropriate envelope functions. In the case of a single non-common atom interface, we simply get

$$
M_{H+L_{-}}^{p q}=\mathrm{i} \frac{a}{8 \sqrt{3}} f_{H_{p}}\left(z_{\mathrm{int}}\right) f_{L_{q}}\left(z_{\mathrm{int}}\right)\left(2 d V_{\mathrm{int}}+\Delta E_{v}\right) .
$$

The optical selection rules associated with heavy and light holes yield a specific polarization for each transition. The most striking effect in the case of non-common atom $\mathrm{QW}$ is the strong $H_{1}-L_{1}$ coupling arising from the different potentials at successive interfaces. A first order calculation gives the polarization rate at $k_{\perp}=0$ of the transition $H_{1}-E_{1}$

$$
P=\frac{\alpha_{\max }-\alpha_{\min }}{\alpha_{\max }+\alpha_{\min }}=\frac{2}{\sqrt{3}} \frac{M_{H_{1} L_{1}}}{\left(L_{1}-H_{1}\right)},
$$

where the matrix element $M_{H_{p} L_{q}}$ is understood to be the sum of the contributions of the two interfaces. Because of sum rules, this polarization becomes almost zero at energies above the $L_{1}-E_{1}$ transition. It is interesting to note that the spectral width of the effect is fixed by the different confinement of heavy and light holes, while its magnitude depends on the weight of envelope functions at the interface, and specific properties of these interfaces. A detailed calculation of the optical spectra can be achieved by introducing the matrix elements $M_{H_{p} L_{q}}$ in a Luttinger Hamiltonian, yielding the correct mixing of states at any $k_{\perp}$, as well as a realistic energy dispersion for each band. It is interesting to notice that although Hamiltonian contains linear in $k_{\perp}$ terms between light holes and heavy holes they do not contribute to the anisotropy when we sum over the different in plane wave vectors. The optical polarization computed for each transition is essentially the same as its value at $k_{\perp}=0$. Therefore, a simplified model such as the diagonal approximation of the valence band dispersion can be used to calculate realistic absorption spectra. This approximation is used in the following section.

\section{QW anisotropy: a non destructive interface characterization}

The main difference between real and ideal quantum wells is that the former do not have nominal growth sequences because of atom exchange and segregation mechanisms, composition overshoots due to transients, etc...Even in the case of the most studied systems, GaAs-AlGaAs, it has been shown that interfaces rather have an exponential "relaxation" profile rather than a composition discontinuity $[17,18]$. This implies that even nominally square, common anion QWs may actually show a small optical anisotropy associated with slightly asymmetric composition profile. Conversely, if anion exchange during the growth interruption which is required to commute four chemical species in any epitaxial system is highly asymmetric, the symmetry of a NCA QW may be of the $D_{2 d}$ type! For instance, let suppose that the anion $\mathrm{A} 1$ is much more stable than $\mathrm{A} 2$ : one may program the nominal growth sequence $\mathrm{C} 1-\mathrm{A} 1-\mathrm{C} 2-\mathrm{A} 2-(\cdots)-\mathrm{C} 2-\mathrm{A} 2-\mathrm{C} 1-\mathrm{A} 1$, and actually get the symmetrical sequence $\mathrm{C} 1-\mathrm{A} 1-\mathrm{C} 2-\mathrm{A} 2-(\cdots)-\mathrm{C} 2-\mathrm{A} 1-\mathrm{C} 1-\mathrm{A} 1$ because the last plane of A2 has been replaced by a plane of A1 before the growth starts again. Polarization resolved spectroscopy associated with proper modeling is a valuable spectroscopic tool to elucidate these phenomena. In general, zero electric field data 
contain the information on the sum of the interface effects, and must be completed with the study of the quantum confined Pockels effect which allows discrimination of the two interface contributions. Needless to mention that polarization-resolved spectroscopy is the subject to many artefacts, especially when searching for polarization rates of the order of $1 \%$. A conventional set-up with careful data analysis gives a typical $0.5 \%$ limit, which can be improved by, at least, one order of magnitude by using polarization modulation.

We illustrate these trends with results obtained in the (InGa)As-InP system which is of special importance because this is the key material for opto-electronics at $1.55 \mu \mathrm{m}$. Photocurrent spectroscopy is a nearly ideal tool in this case. Figure 6 compares the polarization spectra obtained from transmission spectroscopy at $77 \mathrm{~K}$ in (InGa)As-(AlIn)As and (InGa)As-InP QWs having similar quality and similar parameters (nominal well width of $100 \AA$ ). The former sample shows nearly isotropic properties, while the latter shows a "giant" dichroism corresponding to an absorption polarization rate of $10 \%$ between the $H_{1}-E_{1}$ and $L_{1}-E_{1}$ transitions. Corresponding calculations are also shown. The results in (InGa)As-InP QW imply strongly asymmetric interface potentials. A more detailed insight is obtained from the analysis of the QCPE. The polarization spectra at various applied electric fields are shown in Fig. 7, together with the corresponding band-to-band calcula-
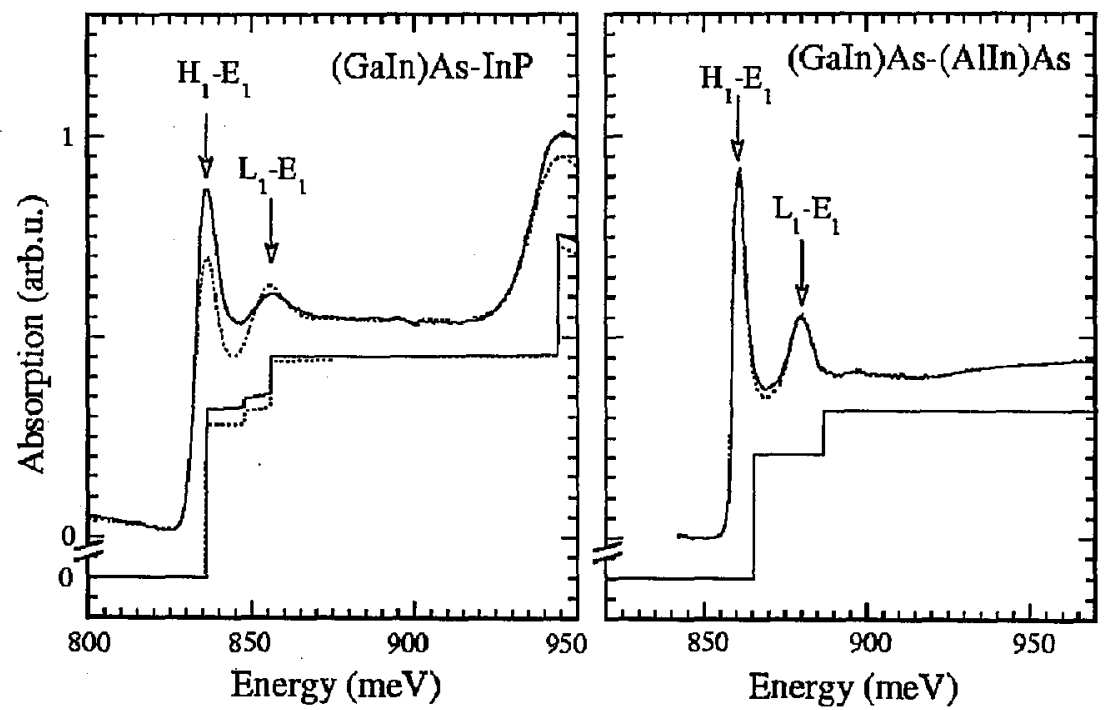

Fig. 6. Polarization-resolved absorption spectra (obtained from transmission spectroscopy at $77 \mathrm{~K}$ ) in nominally $100 \AA$-thick (GaIn)As-InP (left) and (GaIn)As-(AlIn)As (right) $\mathrm{QW}$. The former shows a strong in-plane anisotropy in the spectral range between the $H_{1}-E_{1}$ and $L_{1}-E_{1}$ transitions, while the latter shows essentially isotropic properties. Displayed polarizations are along the [110] and [110] directions, respectively (continuous and dashed lines). The calculated band-to-band spectra (shifted by an arbitrary $7 \mathrm{meV}$ to account for exciton binding energy) are also shown. 


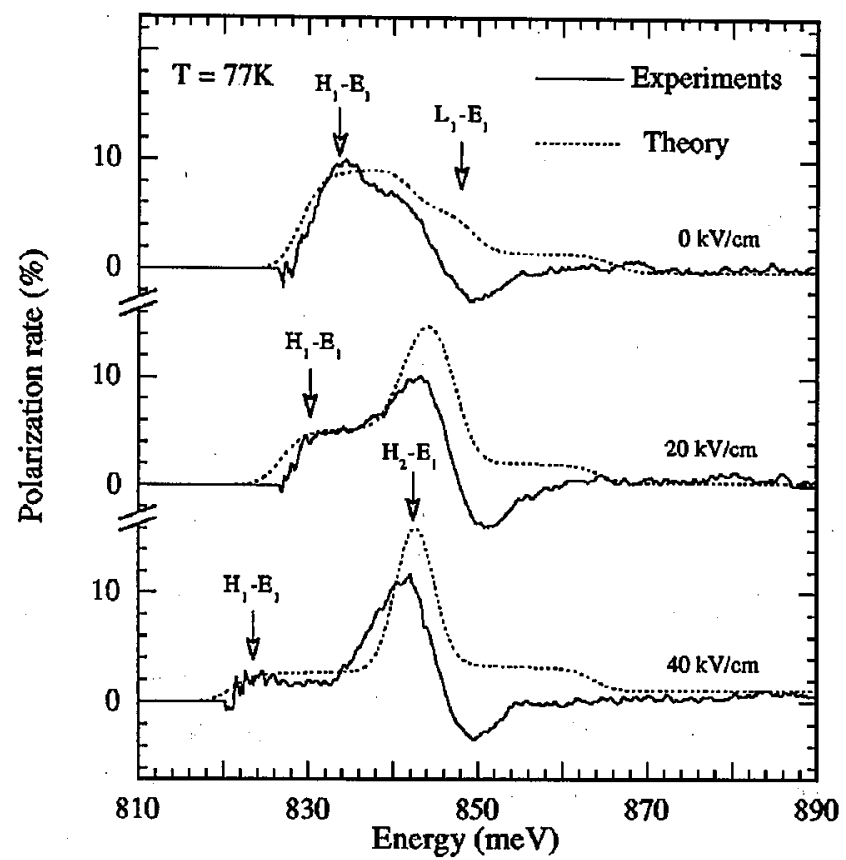

Fig. 7. Electrical field dependency of the absorption polarization rate in the (InGa)As-InP QW of Fig. 6, showing the phenomenology of the "quantum confined Pockels effect". Theoretical curves have been smoothed with a $5 \mathrm{meV}$ width Gaussian and slightly red-shifted for a better comparison with the excitonic contribution. The main discrepancy, which occurs in the $L_{1}-E_{1}$ spectral region, is attributed to the strong coupling between $H_{2}-E_{1}$ and $L_{1}-E_{1}$ excitons which are not taken into account in the model.

tions. The agreement is essentially satisfactory, although the calculation does not include excitons, which obviously contribute to the low temperature spectra in these high quality samples. Excitonic effects influence the QCPE in particular in the vicinity of the $H_{2}-E_{1}$ transition [19]. The dependency of the polarization rate on the well width is illustrated in Fig. 8. Calculations use a similar set of interface potentials for the three samples $\left(d V_{\mathrm{L}} \approx 1.3 \mathrm{eV}, d V_{\mathrm{R}} \approx 0.1 \mathrm{eV}\right)$. These two values have been obtained by fitting the QCPE data. It is finally interesting to compare samples obtained with different growth techniques. In particular, the growth interruption times are in general longer in the case of an ultra-vacuum technique like conventional MBE or gas-source MBE than in atmospheric pressure MOCVD. We show in Fig. 9 the QCPE data obtained in a top-quality (InGa)As-InP quantum well grown by gas-source (GS)-MBE. Nearly zero polarization is observed in flat-band conditions, which indicates that the interfaces are almost symmetrical. Yet, in presence of an electric field, a large polarization appears which implies an interface potential much larger than the band offset. Similar GaAs-AlGaAs or GaInAs-AlInAs wells show nearly no polarization under equivalent polarizations. This indicates that NCA QWs do have specific interface properties, but one 

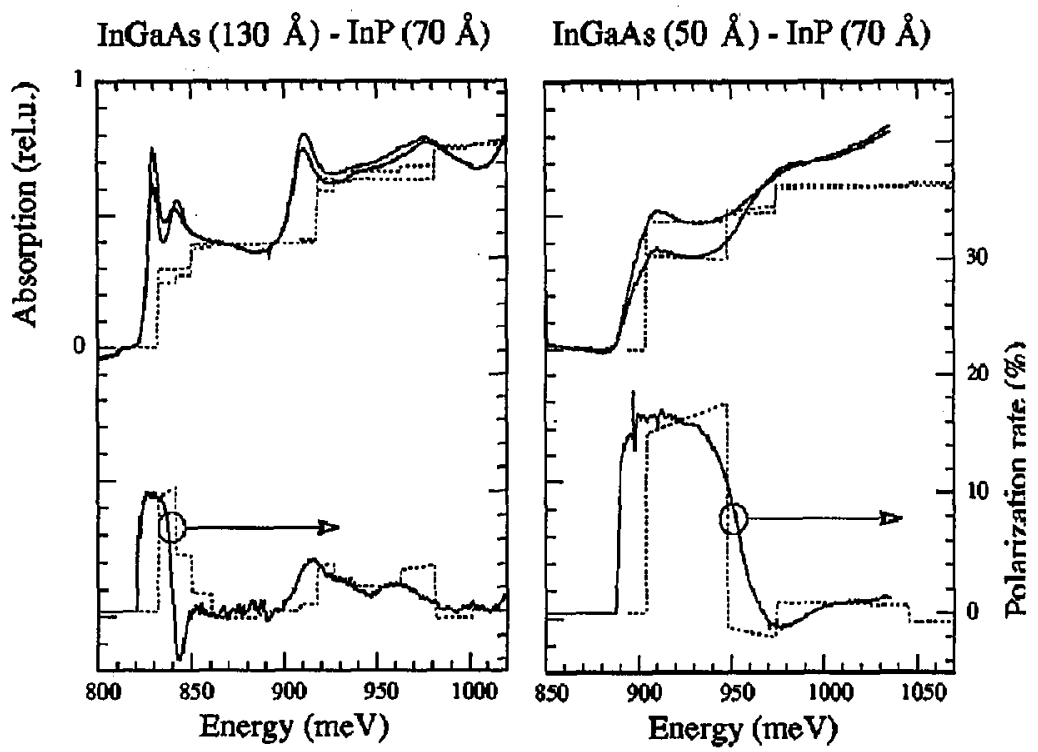

Fig. 8. Polarization- resolved absorption spectra at $77 \mathrm{~K}$ for two MOCVD-grown (In Ga)As-InP QWs showing the well-width dependency of the optical anisotropy. The dashed lines represent the corresponding calculations using a common set of "interface potentials" ( $\left.d V_{\mathrm{L}} \approx 1.3 \mathrm{eV}, d V_{\mathrm{R}} \approx 0.1 \mathrm{eV}\right)$. Discrepancy in the vicinity of the $L_{1}-E_{1}$ transition is attributed to excitonic effect [19].

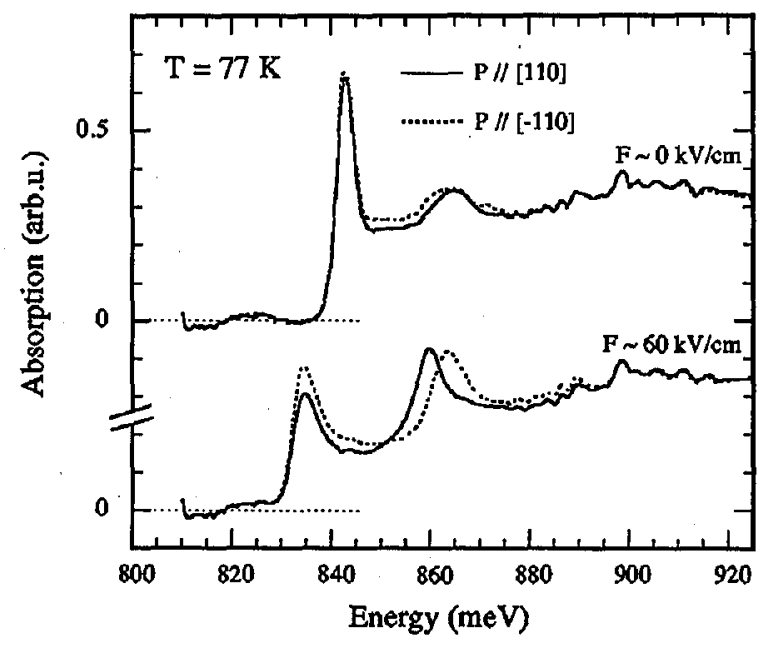

Fig. 9. QCPE-spectra of a $99 \AA$-thick (InGa)As-InP QW grown by gas-source MBE. The zero-field anisotropy is weak ( $D_{2 d}$ symmetry presumably induced by anion exchange), but the large Pockels effect (specifically in the $L_{1}-E_{1}$ region where it is enhanced by the excitonic effect) indicates large interface potentials in this NCA system. 
can act on the overall symmetry of the quantum well with the growth procedure. Equivalent trends have recently been reported in the case of BeTe-ZnSe type-II QWs $[20,21]$.

\section{The effect of chemical intermixing}

Another issue of interest is the role of interface sharpness. We shall discuss first the case of common atom (CA) quantum wells defined by a smooth cation composition profile associated with a valence potential $V(z)$. The physical effect of such a profile is fundamentally different from the effect of an external electric field. From a microscopic point of view, such a valence potential can be represented by steps occurring at each anion plane, while an external field is associated with valence potential steps occurring both at each anion and cation plane. In the first case, the effect of each step in terms of $H-L$ coupling adds up at each 'interface', while in the former, anionic and cationic potential steps cancel each other because of their opposite orientations (see Eq. (17)). Still, because the envelope functions depend on the valence band profile, a $\mathrm{QW}$ with two interfaces of different profiles shall retain a $C_{2 v}$ symmetry, but the changes in terms of $H-L$ coupling are rather small. The corresponding matrix element can be evaluated easily with the following formula:

$$
M_{H_{p} L_{q}}=\mathrm{i} \frac{a}{8 \sqrt{3}} \int f_{H_{p}} f_{L_{q}} \frac{\partial V}{\partial z} \mathrm{~d} z
$$

Again, this formula differs from Eq. (2) of Ref. [3] by a mere factor 2, and the present result does not depend on the choice of unit cell. Obviously, as long as the symmetry of the QW is preserved, no anisotropy is generated. However, in case of segregation profiles which are by construction smooth and asymmetrical, some zero-field anisotropy becomes observable. Numerical estimates for a $100 \AA$-thick GaInAs-AlInAs QW indicate polarization rates in the $1 \%$ range, which are within reach of careful experiments. Hence, polarization-resolved spectroscopy has a very good potential for the non-destructive characterization of buried semiconductor interfaces.

The problem of chemical intermixing of NCA interfaces is rather more difficult to treat because of the separate profiles of the anion and cation composition, and their consequences in terms of local strain. As pointed out earlier in this paper, the essential feature that characterizes a NCA interface is its effective sequence, that is the order in which the anion and cation are changed. This feature remains true in the case of non abrupt interfaces, but a rigorous description of $H-L$ coupling at diffused NCA interfaces is still a challenge.

\section{A parameter free calculation: the interface induced $H_{2}-L_{1}$ anti-crossing}

In a common atom quantum well such as $\mathrm{GaAs}-\mathrm{Al}_{x} \mathrm{Ga}_{1-x} \mathrm{As}$, states with opposite symmetry are coupled even at zero electric field. The interesting point is that such interface couplings can be directly evaluated from the above model (see Eq. (16)) without any adjustable parameter. In the classical envelope function theory, the confinement energies of the $H_{2}$ and $L_{1}$ valence states cross for a given 
well width. This accidental degeneracy is lifted by two separate effects of lowering symmetry; the interface coupling (roto-inversion symmetry breakdown) and the bulk inversion asymmetry, which yields linear in $k_{z}$ terms between heavy and light holes [22]. Since the bulk inversion asymmetry couples only envelopes of opposite parity, it yields no optical anisotropy, but contributes to the anti-crossing between $H_{2}$ and $L_{1}$. The latter coupling has been evaluated by several authors in the literature (it amounts to $0.05 \mathrm{meV}^{\ddagger}$ ), while the former has been omitted by most $\boldsymbol{k} \cdot \boldsymbol{p}$ calculations up to now. We have computed the interface induced anti-crossings at two separate compositions of $\mathrm{Al}, x=0.25$ and $x=1$. The confinement energies as well as the weight of the envelope functions at the interface were evaluated using a four-band envelope function model including spin-orbit. The valence band offset has been taken to be $40 \%$ of the total band gap difference between well and barrier. We obtain respectively an anti-crossings of $0.15 \mathrm{meV}$ and $0.20 \mathrm{meV}$ at very similar well width $(180 \AA)$. Empirical pseudo-potential calculations including spin-orbit coupling give actually similar results [23]. One could be surprised that the effect has only a weak dependence on the composition of the barrier, since the matrix element is directly proportional to the valence band offset. This is actually due to the fact that the weight of the envelope functions at the interface drops when the valence band offset is increased, because of the increased confinement of the holes. Therefore, multiplying the valence band offset by four changes the anti-crossing height by only mere $30 \%$.

\section{Conclusion}

We have discussed recent developments of the physics of rotational symmetry breakdown at semiconductor interfaces, which has quantitatively important influence on the optical properties of non-common atom quantum wells. The present approach brings support (and also, correction of some mistakes) to the heuristic $H_{\mathrm{BF}}$ model that we proposed in 1996 . The new theory bridges the gap between the atomistic theory based on empirical pseudo-potentials and the user-friendly envelope-function approach. Fully $a b$-initio calculations are certainly required to determine theoretically the interface potentials, especially in the case of NCA interfaces, but effective parameters obtained by fitting experimental data can be used for a reliable engineering of anisotropy properties. This recently discovered topic has brought in a critical reexamination of the classical envelope function theory of semiconductor heterostructures which, by construction, misses the intra-cell effect of the composition discontinuity. A completely new linear electro-optical phenomenon, the quantum confined Pockels effect results from the reduced symmetry of interfaces and contributes to a novel type of non-destructive experimental investigation of semiconductor interfaces, based on polarization-resolved spectroscopy.

\section{Acknowledgments}

We would like to acknowledge fruitful discussions and/or collaboration with many colleagues: E.L. Ivchenko, R. Magri, S. Ossicini, D. Bertho, C. Jouanin,

\footnotetext{
$\ddagger$ Using an analytical model for solving the envelope function problem, it can easily be seen that $\left|\left\langle H_{2}\left|C k_{z}\right| L_{1}\right\rangle\right|=8 C / 3 L_{c} \approx 0.05 \mathrm{meV}$, with $L_{c}$ the anti-crossing width and $C \approx 3.4 \mathrm{meV} \AA$ as found in Ref. [22].
} 
J.M. Jancu, L. Vervoort and A. Zunger. We express our gratitude to the epitaxists who have grown the samples used in the experimental part of this work: J.L. Gentner, D. Rondi, J.C. Harmand, J.P. André and F. Mollot.

\section{References}

[1] J.N. Schulman, Y.C. Chang, Phys. Rev. B 31, 2056, 2069 (1985).

[2] O. Krebs, W. Seidel, J.P. André, D. Bertho, C. Jouanin, P. Voisin, Semicond. Sci. Technol. 12, 938 (1997).

[3] O. Krebs, P. Voisin, Phys. Rev. Lett. 77, 1829 (1996).

[4] E.L. Ivchenko, A. Kaminski, U. Rössler, Phys. Rev. B 54, 5852 (1996); E.L. Ivchenko, A. Toropov, P. Voisin, Phys. Solid. State 40, 1748 (1998).

[5] B.A. Foreman, Phys. Rev. Lett. 81, 425 (1998).

[6] R. Magri, unpublished.

[7] J.M. Jancu, private communications and unpublished results.

[8] R. Magri, S. Ossicini, Phys. Rev. B 58, R1742 (1998).

[9] J.C. Slater, G.F. Koster, Phys. Rev. 94, 1498 (1954).

[10] E. Caruthers, P.J. Lin-Chung, Phys. Rev. B 17, 2705 (1978).

[11] J. Khurgin, private communication.

[12] O. Krebs, D. Rondi, J.L. Gentner, L. Goldstein, P. Voisin, Phys. Rev. Lett. 80, 5770 (1998).

[13] B. Foreman, Phys. Rev. Lett. 82, 1339 (1999).

[14] O. Krebs, P. Voisin, Phys. Rev. Lett. 82, 1340 (1999).

[15] S.H. Kwok, H.T. Grahn, K. Ploog, R. Merlin, Phys. Rev. Lett. 69, 973 (1992).

[16] E.L. Ivchenko, A.A. Toropov, P. Voisin, Phys. Solid. State 40, 1748 (1998).

[17] J.M. Moison, C. Guille, F. Barthe, F. Houzay, M. Van-Rompay, Phys. Rev. B 40, 6149 (1989).

[18] J.M. Gérard, J.Y. Marzin, Phys. Rev. B 45, 6313 (1992); J.M. Gérard, Appl. Phys. Lett. 61, 2096 (1992).

[19] A. Toropov, E. Ivchenko, O. Krebs, P. Voisin, to be published.

[20] A.V. Platonov, V.P. Kochereshko, E.L. Ivchenko, G.V. Mikhailov, D.R. Yakovlev, M. Keim, W. Ossau, A. Waag, G. Landwehr, Phys. Rev. Lett. 83, 3546 (1999).

[21] D.R. Yakovlev, E.L. Ivchenko, V.P. Kochereshko, A.V. Platonov, S.V. Zaitsev, A.A. Maksimov, I.I. Tartakovskii, V.D. Kulakovskii, W. Ossau, M. Keim, A. Waag, G. Landwehr, Phys. Rev. B 61, R2421 (2000).

[22] M. Cardona, N.E. Christensen, G. Fasol, Phys. Rev. B 38, 1806 (1988).

[23] R. Magri, private communication. 